\title{
Impacts of skull stripping on construction of three- dimensional T1-weighted imaging-based brain structural network in full-term neonates
}

\section{Geliang Wang}

The First Affiliated Hospital of Xi'an Jiaotong University

Yajie $\mathrm{Hu}$

The First Affiliated Hospital of Xi'an Jiaotong University

\section{Xianjun Li}

The First Affiliated Hospital of Xi'an Jiaotong University

\section{Miaomiao Wang}

The First Affiliated Hospital of Xi'an Jiaotong University

Congcong Liu

The First Affiliated Hospital of Xi'an Jiaotong University

Jian Yang

The First Affiliated Hospital of Xi'an Jiaotong University

Chao Jin ( $\nabla$ chaojin@mail.xjtu.edu.cn )

The First Affiliated Hospital of Xi'an Jiaotong University

\section{Research}

Keywords: Skull stripping, Neonatal brain, brain structural networks, 3D T1 weighted imaging

Posted Date: January 30th, 2020

DOI: https://doi.org/10.21203/rs.2.22268/v1

License: (a) This work is licensed under a Creative Commons Attribution 4.0 International License. Read Full License

Version of Record: A version of this preprint was published at BioMedical Engineering OnLine on June 3rd, 2020. See the published version at https://doi.org/10.1186/s12938-020-00785-0. 


\section{Abstract}

Background: Skull stripping remains a challenge for neonatal brain MR image analysis. However, little is known about how accuracy of the skull stripping affects the neonatal brain tissue segmentation and subsequent network construction. This paper therefore aimed to clarify this issue by comparing two automatic (FSL's Brain Extraction Tool, BET; Infant Brain Extraction and Analysis Toolbox, iBEAT) and a semiautomatic (iBEAT with manual correction) processes in constructing 3D T1-weighted imaging (T1WI)-based brain structural network.

Methods: Twenty-two full-term neonates (gestational age, 37-42 weeks; boys/girls, 13/9) without abnormalities on MRI who underwent brain 3D T1WI were retrospectively recruited. Two automatic (BET and iBEAT) and a semiautomatic preprocessing (iBEAT with manual correction) workflows were separately used to perform the skull stripping. Brain tissue segmentation and volume calculation were performed by a John Hopkins atlas-based method. Sixty-four gray matter regions were selected as nodes; volume covariance network and its properties (clustering coefficient, $C p$; characteristic path length, L $p$; local efficiency, E local ; global efficiency, E global ) were calculated by GRETNA. Analysis of variance (ANOVA) was used to compare the differences in the calculated volumes between three workflows.

Results: There were significant differences in volumes of 48 brain region between three workflows ( $P$ $<0.05)$. Three neonatal brain structural networks presented small-world topology. The semiautomatic workflow showed remarkably decreased $C p$, increased L p, decreased E local, and E global, in contrast to two automatic ones.

Conclusions: Imperfect skull stripping indeed affected the accuracy of brain structural network in fullterm neonates.

\section{Background}

It is known that magnetic resonance imaging (MRI) has become a very important tool for investigating the early brain development and injury in neonates [1,2]. In particular, MRI-based brain structural network analysis provides critical ways to understand the topological structure of brain information integration and transmission during the early development [3-5]. As a basic preprocessing, skull stripping, designed to eliminate skull, scalp, dura, and other non-brain tissues and retain brain parenchyma from brain MRI, is an essential process in brain tissue segmentation and subsequent brain network construction [6]. For neonates, numerous efforts has been made to perform the automated processing [7-13], such as FSL's brain extraction tool (BET) [14] and infant brain extraction and analysis toolbox (iBEAT developed by the IDEA group at the University of North Carolina at Chapel Hill) [15]. However, skull stripping remains a challenge for neonatal brain MRI analysis due to its low tissue contrast, large within-tissue intensity variations, and regionally heterogeneous [16]. Besides, inaccurate skull stripping, e.g. unremoved nonbrain tissues would result in the overestimation of local brain volume and cortical thickness [17]. And it would further affect the construction of brain structural network. To our knowledge, little is known about 
the impact of preprocessing accuracy on the accuracy of brain tissue segmentation and structural network construction.

The present study aimed to investigate the effects of skull stripping on brain tissue segmentation and structural network construction based on three-dimensional (3D) T1-weighted imaging (T1WI). Firstly, the study constructed the processing flow based on 3D T1-weighted MRI on 22 term neonates' gray matter; secondly, we did the repeatability and consistency of brain region volume's segmentation were further performed to verify the accuracy; in final part, the study correlated the 64 brain regions volumes of the other two workflows, which did a comparison on three workflows' with the standard volume. Such a workflow could be apply applied to characterize structural connectivity and may provide valuable anticipatory information about the potential for encountering abnormalities at a later stage in development.

\section{Results}

\section{Repeatability and Reliability}

The Bland-Altman graph of two repeated measurements presents that $95 \%$ of the differences between two measurements located in the mean $\pm 1.96 \mathrm{SD}$ range that suggest the good repeatability (Fig. 1A). Meanwhile, the ICC analysis indicates the average correlation coefficient was 0.894 . Most of values were greater than 0.8, a few brain regions's (caudate nucleus, precuneus, superior occipital gyrus, and inferior occipital gyrus) correlation coefficient were relatively lower, but there were greater than 0.7 (Fig. 1B).

\section{Comparisons of brain structural network between three workflows}

Brain region volume

The volume of 64 brain regions difference did the ANOVA analysis among three workflows which are shown below (Fig. 2). From figure, we can see that the volume of 50 brain regions have significant difference. Meanwhile, there was no significant difference the volume of 14 brain regions.

\section{Small-world properties}

In the defined threshold range, the neonatal brain network exhibited high-efficiency small-world topology (Fig. 3).

\section{Properties of brain structural network}

The corrected workflow, compared with the others, showed a significantly decreased $\mathrm{C}_{p}$ and increased $\mathrm{L}_{p}$. With regard to network efficiency, the corrected workflow showed a significantly decreased $\mathrm{E}_{\text {local }}$ and 
$E_{\text {global }}$ (Fig. 4).

\section{Discussion}

To investigate the impacts of skull stripping on the accuracy of brain tissue segmentation and structural network construction, three workflows (BET, iBEAT and iBEAT with manual correction) were compare to perform the 3D T1WI-based brain structural network. Our results indicated that the iBEAT with manual correction showed a more accurate consistency and repeatability in brain segmentation. Besides, significant differences in calculations of brain volume and structural network properties between the three workflows further implied the importance of accurate skull stripping in brain segmentation and subsequent brain network construction.

The ICC results indicated that the majority of brain regions showed good repeatability and reliability, while the ICC of some regions, such as caudate nucleus, gyrus rectus, left superior parietal gyrus, left precuneus, left superior occipital gyrus and right inferior occipital gyrus, were relatively lower. One reason may be rooted in the inherently low spatial resolution, insufficient tissue contrast, and ambiguous tissue intensity distributions in neonatal MRI [16]. One the other, the adult head coil used in this study may affect the MR image quality in neonates $[18,19]$. In this regard, MRI acquisition settings should be adjusted for neonates.

By comparing the three workflows, we found that the slight adjustment for skull stripping would remarkably affect the calculations of regional brain volume. Of 64 regions, significant differences existed in brain volume calculations of 50 regions between the three workflows. This further confirmed the difficulty of brain segmentation in neonatal MRI. Specifically, the most brain volumes calculated by BET workflow were smaller than those by iBEAT and iBEAT with manual correction. This may be linked with the difference of skull stripping in neonatal MRI between BET and iBEAT [16]. It is noting that more unremoved skull components were found in BET than iBEAT; while unremoved components by iBEAT mainly located in the base of skull. It may be such facts that led to the larger brain volumes by iBEAT than iBEAT with manual correction. From the above, the accuracy of skull stripping would have considerable effects on the neonatal brain segmentation.

By studying the brain structural network, we found the highly small-world topology in full-term neonates. This may suggest that a highly efficient brain network, serving for brain information integration and transfer has been constructed in the early development. This is also in agreement with prior findings in neonates [5]. Besides, significant differences were found in local $\left(\mathrm{C}_{p}\right.$ and $\mathrm{E}_{\text {loc }}$ and global $\left(\mathrm{E}_{\text {glob }}\right)$ topological properties of brain structural network between the three workflows. These may indicate that these parameters were sensitive to the accuracy of preprocessing (skull stripping).

The study had several limitations. First, the sample size is relatively small in this study, and more data should been acquired to further improve the accuracy. Second, the template and atlas we used are made by foreign neonates, so to some extent, it would not be appropriate for the Chinese neonates. Atlas and 
the image being classified can lead to large errors in the initial estimate of tissue intensity distributions and require large deformations from the registration phase, so further study is needed to develop a dedicated atlas and template apply to Chinese neonates.

\section{Conclusions}

In this study, we quantitatively analyzed the influences of skull stripping on brain volume calculation and topological properties of brain structural network in neonates. Our results indicated that although the brain networks had robust small-world configuration, there were significant differences in both local and global topological parameters between the three workflows. These further enhanced the importance of accurate skull stripping in calculations of brain tissue volume and brain structural network construction.

\section{Methods}

This study was approved by the local institutional review board of the first author's affiliation. The parents of the neonates were informed regarding the goals and risks of the MRI scan, and requested for the written consent.

\section{Subjects}

This study recruited 22 full-term neonates (13 males and 9 females; gestational age range: $37-42$ weeks) without any MRI abnormalities or evidences of any clinical episodes that might cause cerebral damages.

\section{MRI data acquisition}

All data were acquired on a 3.0 Tesla scanner (Signa HDxt, General Electric Medical System, Milwaukee, WI, USA) with an 8-channel phase array radio-frequency head coil. To reduce the head movement and complete the MRI procedure, the subjects were sedated with a relatively low dose of oral chloral hydrate $(25-50 \mathrm{mg} / \mathrm{kg})$. The potential risks of the chloral hydrate were fully considered. The selection, monitoring, and management of subject were strictly performed following the guidelines [20]. Neonates were laid in a supine position and snugly swaddled in blankets. A pediatrician was present during the MRI scan. Micro earplugs were inserted into the external auditory canal for hearing protection. Heads of the subjects were immobilized by molded foam, which was placed around the head. The temperature, heart rate, and oxygen saturation were monitored throughout the procedure.

Three-dimensional fast spoiled gradient-recalled echo (3D-FSPGR) T1-weighted magnetic resonance images were acquired with the parameters: repetition time/echo time $=10.28 \mathrm{~ms} / 4.62 \mathrm{~ms}$, inversion time $=400 \mathrm{~ms}, \mathrm{FOV}=240 \mathrm{~mm}$; voxel size $=0.94 \times 0.94 \times 1 \mathrm{~mm}^{3}$. 


\section{Construction of brain structural network}

Data preprocessing. To investigate the impacts of data preprocessing on network construction, three preprocessing workflows were performed by BET, iBEAT and iBEAT with manual correction.

BET workflow: non-brain tissues in each T1WI image were automatically removed by BET. iBEAT workflow: three automatic preprocessing steps, including brain contrast enhancement and skull stripping were sequentially performed. iBEAT with manual correction: (1) manually align the AC-PC line; (2) strip the skull by using iBEAT; (3) manually remove the remaining non-brain tissues.

Calculations of brain region volume. To calculate the volume of each brain volume, the preprocessed data were firstly registered to a standard 3D-T1WI-based template (Johns Hopkins University) by linear (rigid transformation) and nonlinear (affine transformation) registrations. And then the volume of each brain region can be estimated by Eq.(1):

$$
V_{i}=\frac{\sum_{V_{t}} J_{F}}{\left|A_{3 \times 3}^{\text {ixid }}\right|\left|A_{3 \times 3}^{\text {afine }}\right|}
$$

where, $V_{i}$ is the brain volume of the $i$ th region of individual subjects, $V_{t}$ represents the corresponding

area in the template to accumulates. $\left|\mathrm{A}_{3 \times 3}^{\text {rigid }}\right|$ is the determinant of $3 \times 3$ sub-matrix in upper left corner of

rigid transformation matrix, and $\left|\mathrm{A}_{3 \times 3}^{\text {affine }}\right|$ is the determinant of $3 \times 3$ sub-matrix in upper left corner of

affine transformation matrix.

Construction of brain structural network. By using the graph theoretic approaches, the cortical and subcortical regions were used as nodes to construct the brain networks, with connections between nodes defined as correlations between regional brain volumes. Here, 64 brain regions that mainly involve the gray matters and several important subcortical regions (thalamus, hippocampus and cerebellum) were selected as network nodes (Table 1). GRETNA (www.nitrc.org/projects/gretna/) was used to construct the network. Partial correlations between all nodes' volumes were firstly estimated as edges of the network, and then network was constructed by a binary connection matrix. The network properties, such as

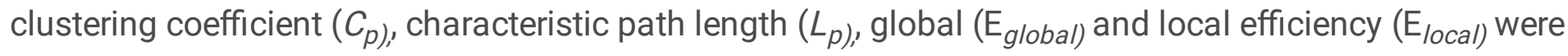
calculated. The $\mathrm{C}_{p}$ of a network is defined by the average of the clustering coefficients across nodes, where the $\mathrm{C}_{p}$ of a node is the ratio of the number of actually connection nearest neighbors of this node to the maximum number of possible connection [21]. $C_{p}$ quantifies the local interconnectivity of a graph. The $L_{p}$ of a graph is the average of the shortest path length between all pairs of nodes in the network, and it is an indicator of overall routing efficiency of a graph [22]. The $E_{\text {local }}$ of a network is the average of the local efficiency over all nodes. It measures the mean local efficiency of the network. The $\mathrm{E}_{\text {global }}$ of a network is defned by the mean shortest path length [23]. It measures the extent of information propagation through the whole network. Typically, a small-world network should fulfill the following conditions: $\mathrm{C}_{p} \mathrm{C}_{\text {rand }}>1$ and $\mathrm{L}_{p} \mathrm{~L}_{\text {rand }} \approx 1$. 


\section{Statistical analysis}

Given the manual corrections for AC-PC alignment and skull stripping, the repeatability and consistency of brain volume calculations between two repeated measurements were evaluated by the Bland-Altman graph and intraclass correlation coefficient (ICC). Analysis of variance (ANOVA) was used to compare the differences in the calculated volume of 64 brain regions between the three workflows.

All the segmentation, calculation of brain region volume and network parameters and statistical analysis were performed by using the MATLAB R2016b (Mathworks Inc, Natick, MA, USA).

\section{References}

1. Dubois J, Dehaene-Lambertz G, Kulikova S, Poupon C, Hüppi PS, Hertz-Pannier L. The early development of brain white matter: a review of imaging studies in fetuses, newborns and infants. Neuroscience. 2014;276: 48-71.

2. Holland D, Chang L, Ernst TM, Curran M, Buchthal SD, Alicata D, Skranes J, Johansen H, Hernandez A, Yamakawa R, Kuperman JM, Dale AM. Structural growth trajectories and rates of change in the first 3 months of infant brain development. JAMA Neurol. 2014;71(10):1266-74.

3. Cao $\mathrm{M}$, Huang $\mathrm{H}, \mathrm{He}$ Y. Developmental connectomics from infancy through early childhood. Trends Neurosci. 2017;40(8):494-506.

4. Gao W, Lin W, Grewen K, Gilmore JH. Functional connectivity of the infant human brain: plastic and modifiable. Neuroscientist. 2017;23(2):169-184.

5. Brown CJ, Miller SP, Booth BG, Andrews S, Chau V, Poskitt KJ, Hamarneh G. Structural network analysis of brain development in young preterm neonates. Neuroimage. 2014;101:667-80.

6. Huang H, Shu N, Mishra V, Jeon T, Chalak L, Wang ZJ, Rollins N, Gong G, Cheng H, Peng Y, Dong Q, He Y. Development of human brain structural networks through infancy and childhood. Cereb Cortex. 2015;25(5):1389-404.

7. Weisenfeld NI, Warfield SK. Automatic segmentation of newborn brain MRI. Neuroimage. 2009;47 (2):564-572

8. Shi F, Fan Y, Tang S, Gilmore JH, Lin W, Shen D. Neonatal brain image segmentation in longitudinal MRI studies. Neuroimage. 2010;49 (1):391-400

9. Gui L, Lisowski R, Faundez T, Hüppi PS, Lazeyras F, Kocher M. Morphology-driven automatic segmentation of MR images of the neonatal brain. Med Image Anal. 2012;16(8):1565-79.

10. Mahapatra D. Skull stripping of neonatal brain MRI: using prior shape information with graph cuts. J Digit Imaging. 2012;25(6):802-14.

11. Anbeek P, Išgum I, van Kooij BJ, Mol CP, Kersbergen KJ, Groenendaal F, Viergever MA, de Vries LS, Benders MJ. Automatic segmentation of eight tissue classes in neonatal brain MRI. Plos One. 2013;8(12):e81895. 
12. Gousias IS, Alexander H, Counsell SJ, Latha S, Rutherford MA, Heckemann RA, Hajnal JV, Daniel R, David EA. Magnetic resonance imaging of the newborn brain: automatic segmentation of brain images into 50 anatomical regions. Plos One. 2013;8(4):e59990

13. Cardoso MJ, Melbourne A, Kendall GS, Modat M, Robertson NJ, Marlow N, Ourselin S. AdaPT: An adaptive preterm segmentation algorithm for neonatal brain MRI. Neuroimage. 2013;65(1):97-108

14. Smith SM. Fast robust automated brain extraction. Hum Brain Mapp. 2002;17(3):143-155.

15. Dai Y, Shi F, Wang L, Wu G, Shen D. iBEAT: a toolbox for infant brain magnetic resonance image processing. Neuroinformatics. 2013;11:211-225.

16. Li G, Wang L, Yap PT, Wang F, Wu Z, Meng Y, Dong P, Kim J, Shi F, Rekik I, Lin W, Shen D. Computational neuroanatomy of baby brains: A review. Neuroimage. 2019;185:906-925.

17. Shi F, Wang L, Dai Y, Gilmore JH, Lin W, Shen D. LABEL: pediatric brain extraction using learningbased meta-algorithm. Neuroimage. 2012;62 (3):1975

18. Hillenbrand CM, Reykowski A. MR imaging of the newborn: a technical perspective. Magn Reson Imaging Clin N Am. 2012;20(1):63-79.

19. Hughes EJ, Winchman T, Padormo F, Teixeira R, Wurie J, Sharma M, Fox M, Hutter J, Cordero-Grande L, Price AN, Allsop J, Bueno-Conde J, Tusor N, Arichi T, Edwards AD, Rutherford MA, Counsell SJ, Hajnal JV. A dedicated neonatal brain imaging system. Magn Reson Med. 2017;78(2):794-804.

20. Coté CJ, Wilson S. Guidelines for monitoring and management of pediatric patients before, during, and after sedation for diagnostic and therapeutic procedures. Pediatr Dent. 2019;41(4):26E-52E.

21. Watts DJ, Strogatz SH. Collective dynamics of 'small-world' networks. Nature. 1998;393(6684):4402.

22. Rubinov M, Sporns 0 . Complex network measures of brain connectivity: Uses and interpretations. Neuroimage. 2010;52(3):1059-1069

23. Latora V, Marchiori M. Efficient behavior of small-world networks. Phys Rev Lett. 2001;87(19):198701

\section{Declarations}

\section{Ethics approval and consent to participate}

All procedures performed in studies involving human participants were in accordance with the ethical standards of the institutional and/or national research committee and with the 1964 Helsinki declaration and its later amendments or comparable ethical standards. Informed consent was obtained from all individual participants included in the study.

\section{Consent for publication}

All authors confirmed the consent for publication.

\section{Availability of data and material}


The codes and datasets of this study are available from the corresponding authors upon reasonable request.

\section{Competing interests}

The authors declare that they have no conflict of interest.

\section{Funding}

This study was funded by National Natural Science Foundation of China (No. 51706178, 81171317, 81971581), National Key Research and Development Program of China (2016YFC0100300), the 2011 New Century Excellent Talent Support Plan of the Ministry of Education, China (NCET-11-0438).

\section{Authors' contributions}

G.L.W. and Y.J.H. performed the data acquisition, analysis and manuscript writing. X.J.L. performed the data processing method, M.M.W. and C.C.L. performed the data acquisition, J.Y. and C.J. designed the study, analyzed the data and wrote the manuscript.

\section{Acknowledgements}

The authors appreciated Prof. Li Liu, Prof. Xihui Zhou, Dr. Hongxia Song and Gailian Li from the Neonatology Department for preparing and monitoring the neonates before and during imaging. We also thank all participants and their parents for their loyalty and cooperation.

\section{Table}

Table 1 The list of 64 brain regions 


\begin{tabular}{|c|c|c|c|c|c|}
\hline No. & Region & Hemisphere & No. & Region & Hemisphere \\
\hline 1 & thalamus & left & 33 & angular gyrus & left \\
\hline 2 & thalamus & right & 34 & angular gyrus & right \\
\hline 3 & putamen & left & 35 & superior temporal gyrus & left \\
\hline 4 & putamen & right & 36 & superior temporal gyrus & right \\
\hline 5 & globus pallidus & left & 37 & middle temporal gyrus & left \\
\hline 6 & globus pallidus & right & 38 & middle temporal gyrus & right \\
\hline 7 & caudate nucleus & left & 39 & inferior temporal gyrus & left \\
\hline 8 & caudate nucleus & right & 40 & inferior temporal gyrus & right \\
\hline 9 & superior frontal gyrus & left & 41 & fusiform gyrus & left \\
\hline 10 & superior frontal gyrus & right & 42 & fusiform gyrus & right \\
\hline 11 & middle frontal gyrus & left & 43 & parahippocampal gyrus & left \\
\hline 12 & middle frontal gyrus & right & 44 & parahippocampal gyrus & right \\
\hline 13 & inferior frontal gyrus & left & 45 & entrhinal cortex & left \\
\hline 14 & inferior frontal gyrus & right & 46 & entrhinal cortex & right \\
\hline 15 & medial fronto-orbaital gyrus & left & 47 & superior occipital gyrus & left \\
\hline 16 & medial fronto-orbaital gyrus & right & 48 & superior occipital gyrus & right \\
\hline 17 & lateral fronto-orbital gyrus & left & 49 & middle occipital gyrus & left \\
\hline 18 & lateral fronto-orbital gyrus & right & 50 & middle occipital gyrus & right \\
\hline 19 & gyrus rectus & left & 51 & inferior occipital gyrus & left \\
\hline 20 & gyrus rectus & right & 52 & inferior occipital gyrus & right \\
\hline 21 & precentral gyrus & left & 53 & cuneus & left \\
\hline 22 & precentral gyrus & right & 54 & cuneus & right \\
\hline 23 & postcentral gyrus & left & 55 & lyngual gyrus & left \\
\hline 24 & postcentral gyrus & right & 56 & lyngual gyrus & right \\
\hline 25 & superior parietal gyrus & left & 57 & amygdala & left \\
\hline 26 & superior parietal gyrus & right & 58 & amygdala & right \\
\hline 27 & precuneus & left & 59 & hippocampus & left \\
\hline 28 & precuneus & right & 60 & hippocampus & right \\
\hline
\end{tabular}




\begin{tabular}{|llllll|}
29 & cingular gyrus & left & 61 & cerebellar hemisphere & left \\
30 & cingular gyrus & right & 62 & cerebellar hemisphere & right \\
31 & supramarginal gyrus & left & 63 & insular cortex & left \\
32 & supramarginal gyrus & right & 64 & insular cortex & right \\
\hline
\end{tabular}

\section{Figures}

(A)

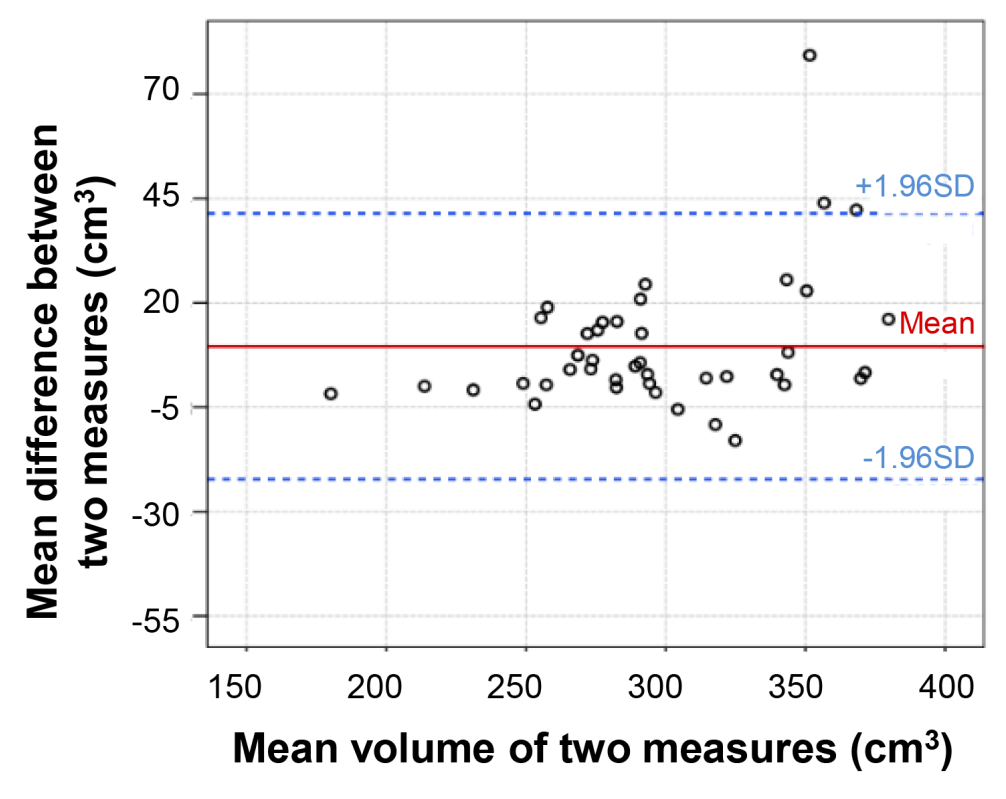

(B)

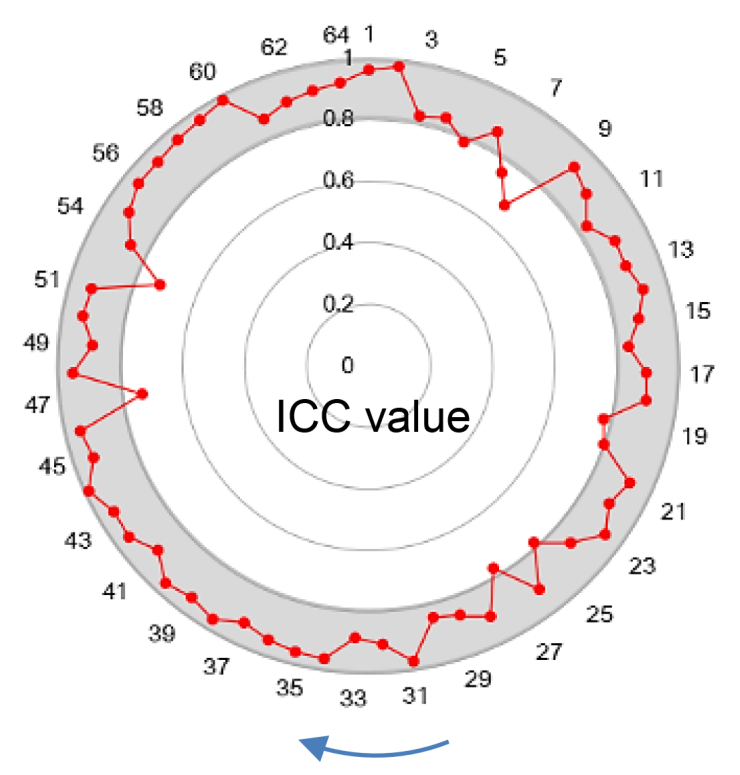

64 brain regions

Figure 1

Results of repeatability and reliability of brain region volume calculated by iBEAT with correction. (A) Bland-Altman graph of whole brain volume between two measurements; (B) ICC of volume of 64 brain regions between two measurements. 
$* P<0.05$

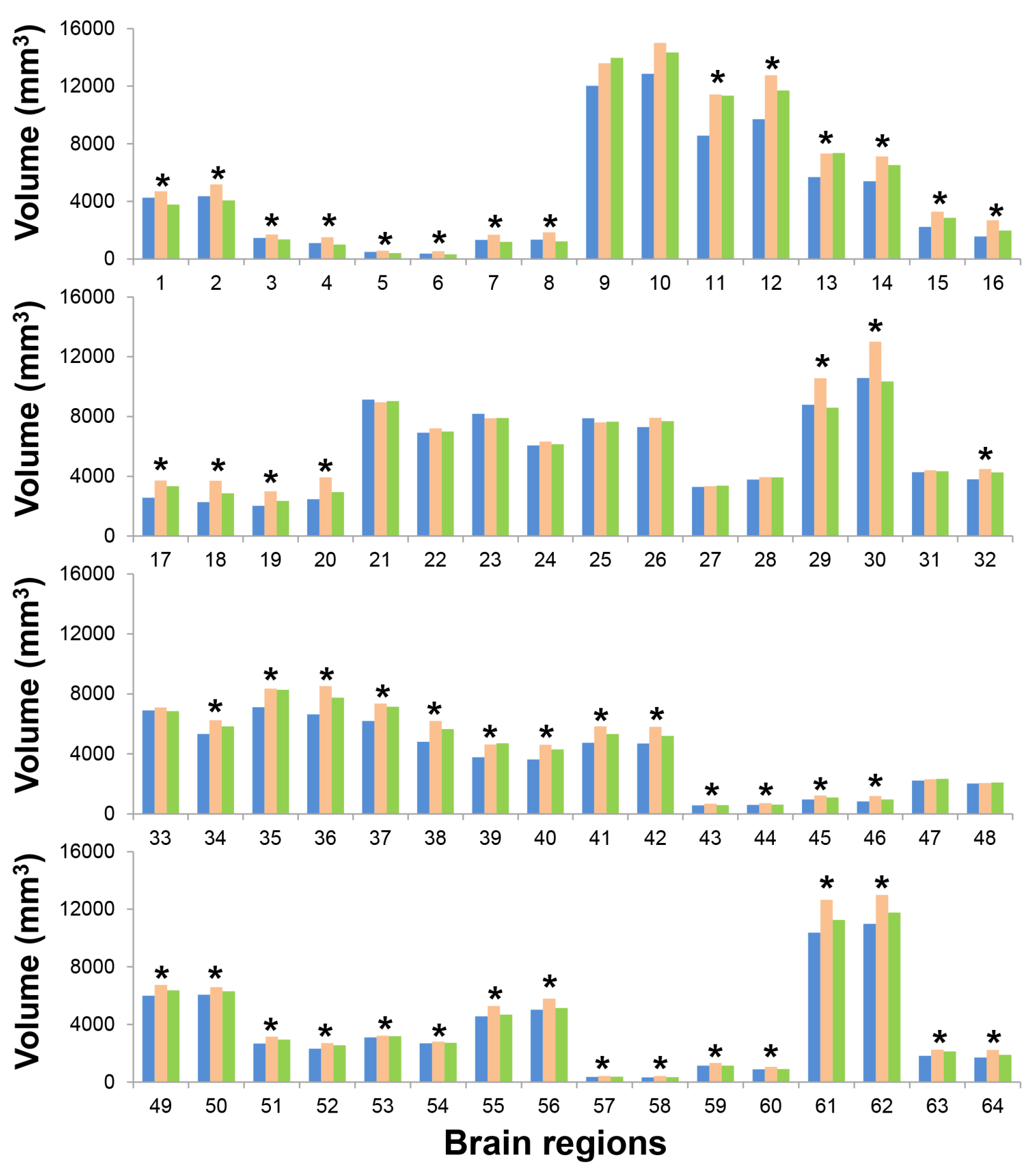

Figure 2

Comparisons of the calculated volume of 64 brain regions between BET, iBEAT and iBEAT with correction. 


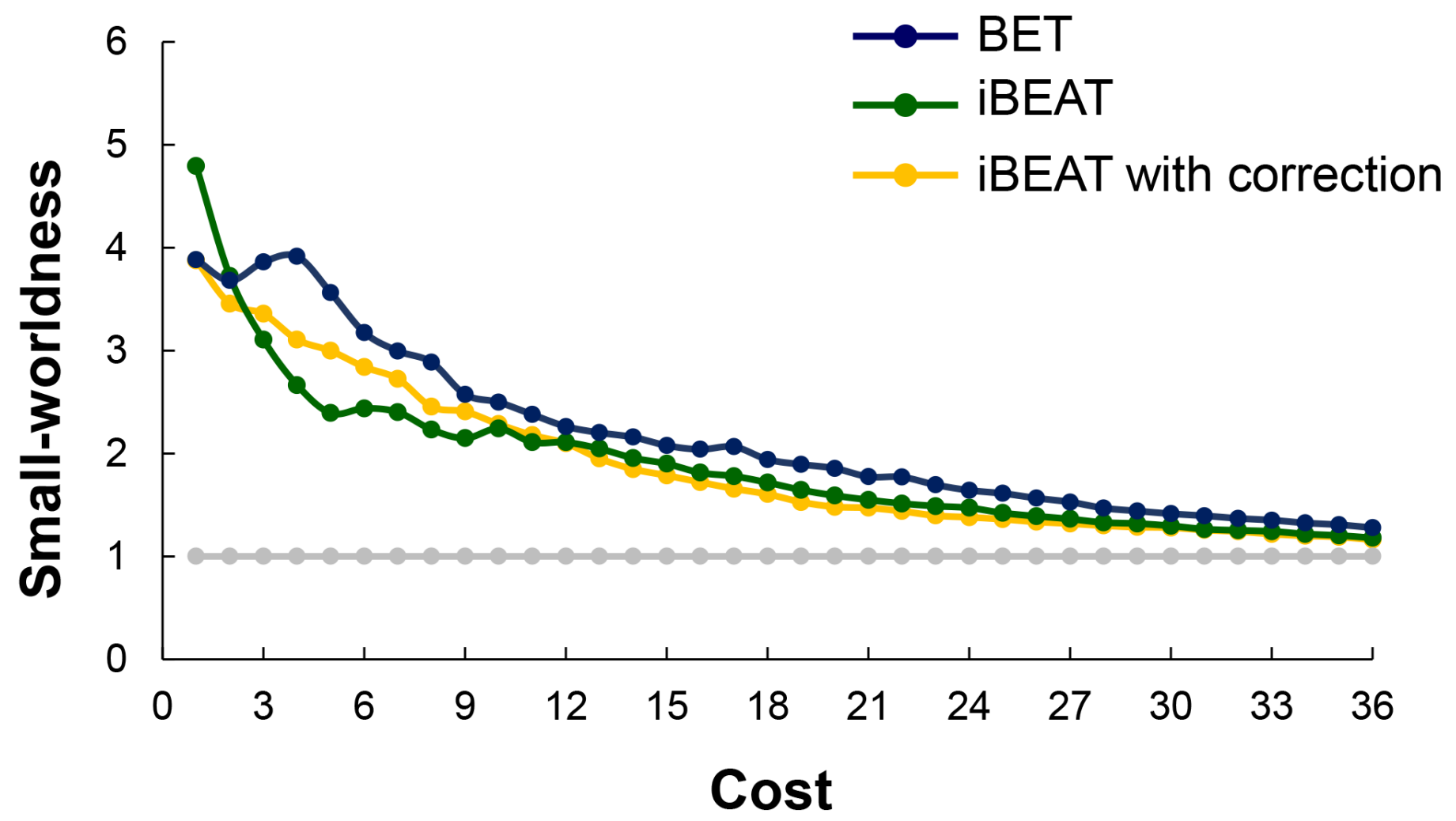

Figure 3

Comparisons of small-world property of brain structural network between BET, iBEAT and iBEAT with correction. 
$\rightarrow$ BET

(A)
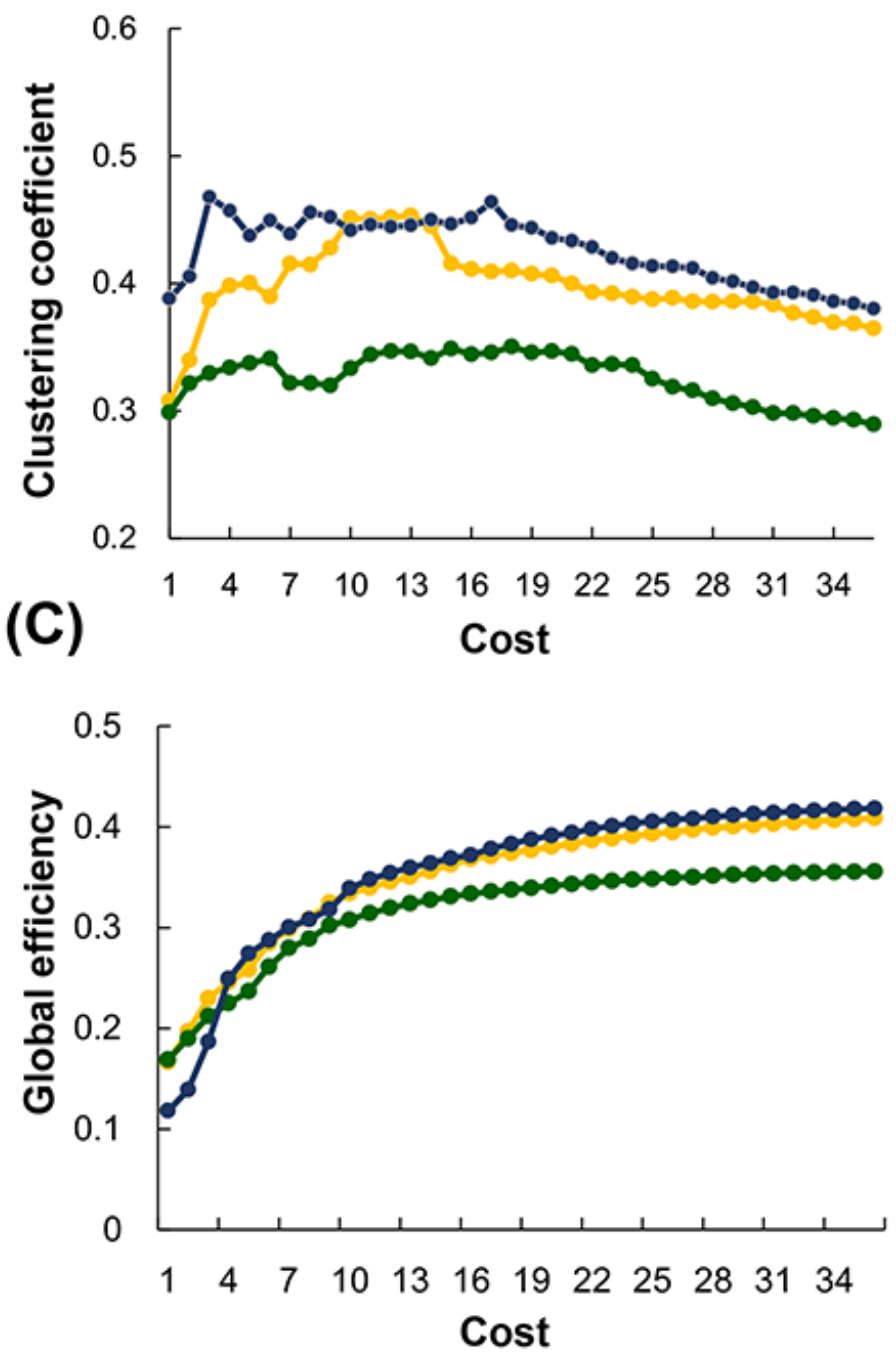

$\multimap$ iBEAT with correction

(B)
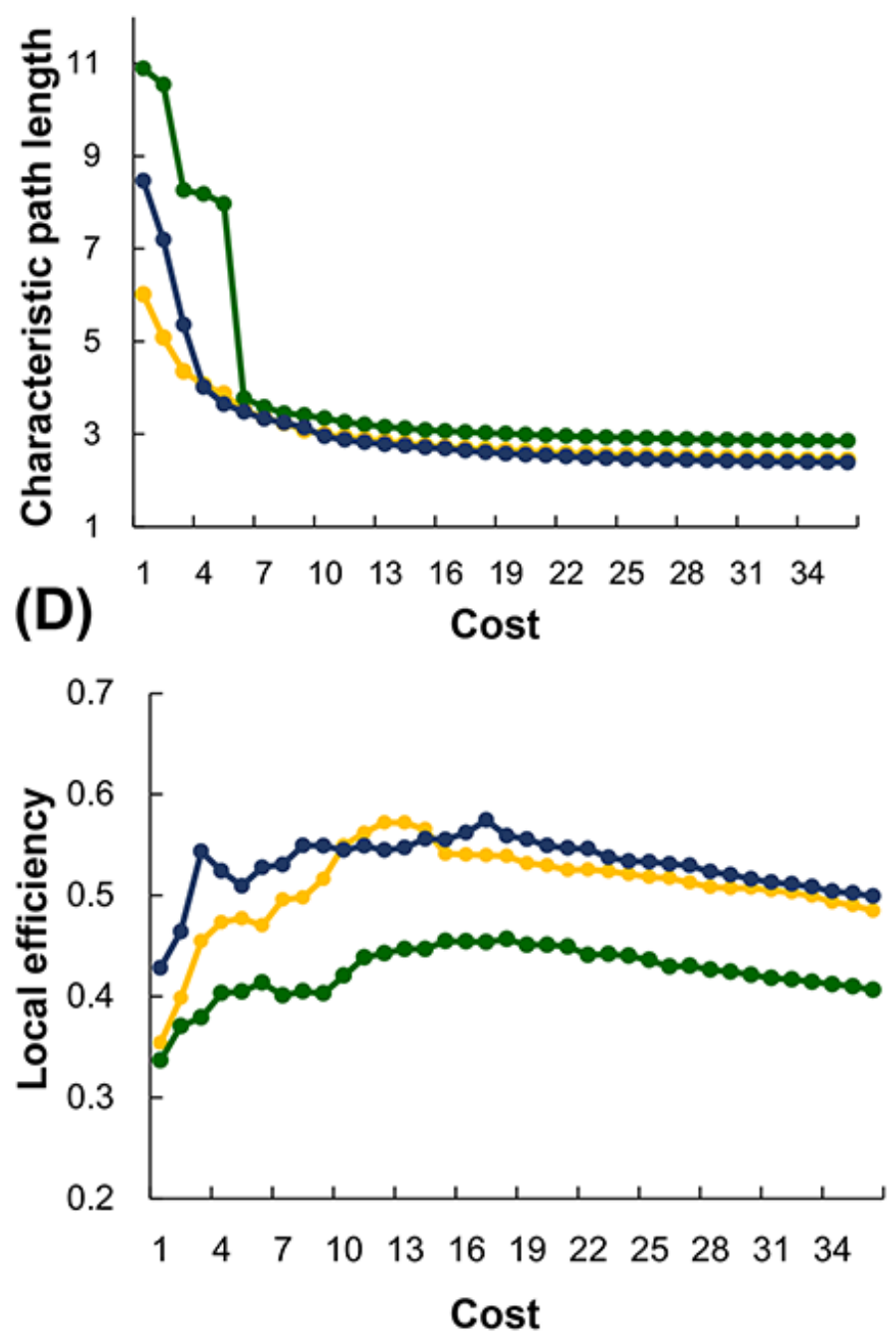

Figure 4

Comparisons of properties of brain structural network between BET, iBEAT and iBEAT with correction. (A) clustering coefficient, (B) characteristic path length, (C) global efficiency, (D) local efficiency. 


\section{BET}

iBEAT

iBEAT with

\section{correction}
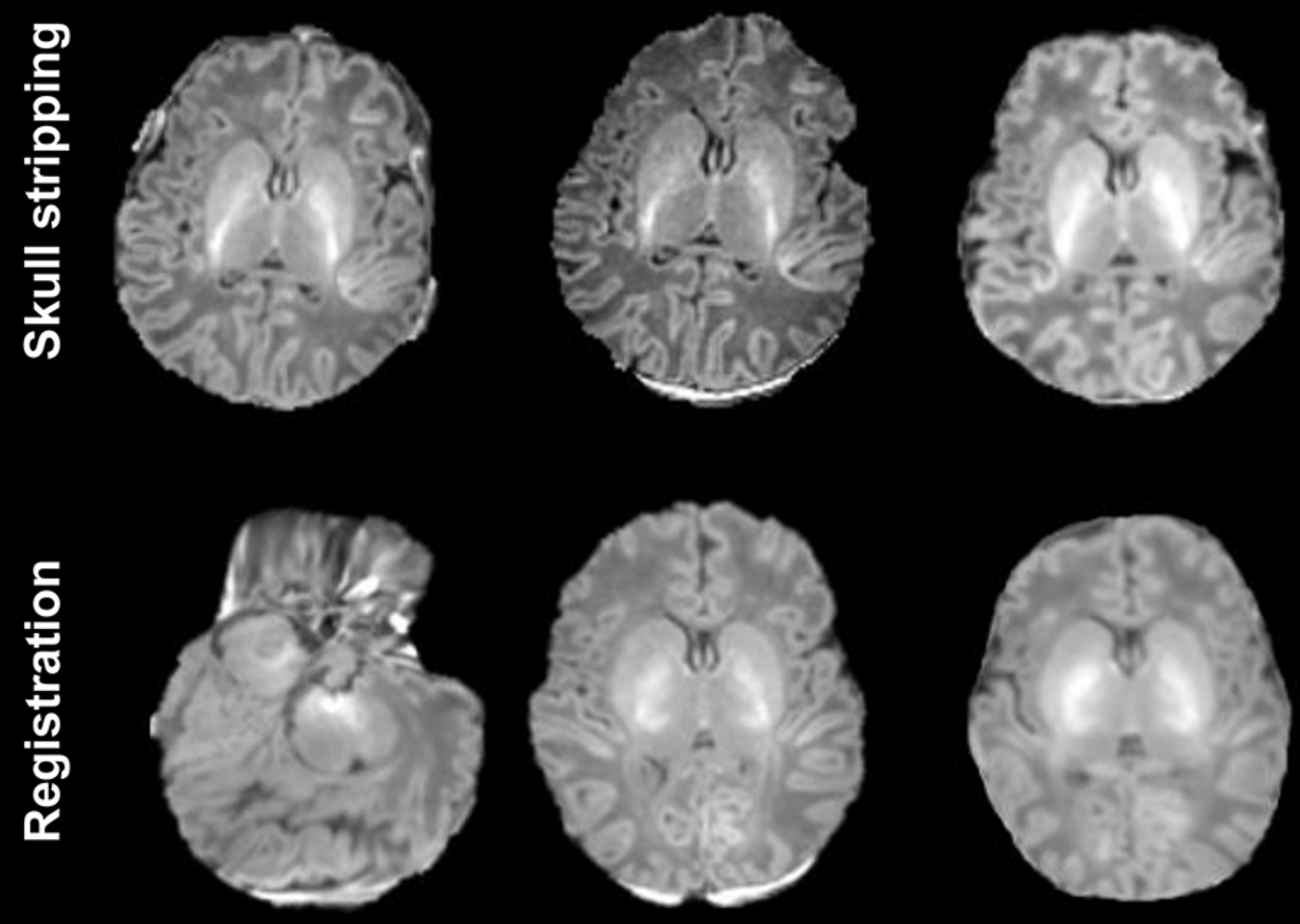

\section{Figure 5}

Illustrations of skull stripping and registration by BET, iBEAT and iBEAT with manual correction. 